\title{
Reversible Language Extensions and their Application in Debugging
}

\author{
Zoé Drey ${ }^{1}$, José F. Morales ${ }^{1}$, and Manuel V. Hermenegildo ${ }^{2,1}$ \\ ${ }^{1}$ IMDEA Software Institute, Madrid (Spain) \\ ${ }^{2}$ School of Computer Science, T. U. Madrid (UPM), (Spain)
}

\begin{abstract}
A range of methodologies and techniques are available to guide the design and implementation of language extensions and domainspecific languages. A simple yet powerful technique is based on source-tosource transformations interleaved across the compilation passes of a base language. Despite being a successful approach, it has the main drawback that the input source code is lost in the process. When considering the whole workflow of program development (warning and error reporting, debugging, or even program analysis), program translations are no more powerful than a glorified macro language. In this paper, we propose an augmented approach to language extensions for Prolog, where symbolic annotations are included in the target program. These annotations allow selectively reversing the translated code. We illustrate the approach by showing that coupling it with minimal extensions to a generic Prolog debugger allows us to provide users with a familiar, source-level view during the debugging of programs which use a variety of language extensions, such as functional notation, DCGs, or CLP $\{Q, R\}$.
\end{abstract}

Keywords: language extensions, debuggers, logic programming, constraint programming

\section{Introduction}

One of the key decisions when specifying a problem or writing a program to solve it is choosing the right language. Even when using recent high-level and multiparadigm languages, the programmer often still needs precise, domain-specific vocabulary, notations, and abstractions which are usually not readily available. These needs are the main motivation behind the development of domain-specific languages, which enable domain experts to express their solutions in terms of the most appropriate constructs.

However, designing a new language can be an intimidating task. A range of methodologies and tools have been developed over the years in order to simplify this process, from compiler-compilers to visual environments [12]. A simple, yet powerful technique for the implementation of domain-specific languages is based on source-to-source transformations. Although in this process the source and target language can be completely different, it is frequent to be just interested 
in some idiomatic extensions, i.e., adding domain specific features to a host language while preserving the availability of most of the facilities of this language. Examples of such extensions are adding functional notation to a language that does not support it, adding a special notation for grammars (such as Definite Clause Grammars (DCGs) [15]), etc. Such transformations have been proposed in the context of object-oriented programming (e.g.,for Java, [14]), functional programming (e.g.,for Haskell, [9]), or logic programming (the term_expansion facility in most Prologs, or the extended mechanisms of [2,8]) In this approach, the language implementations provide a collection of hooks that allow the programmer to extend the compiler and implement both syntactic and semantic variations.

An important practical aspect is that, in addition to appropriate notation, the programmer also needs environments that help during program development. In particular, basic tools such as editors, analyzers, and, specially, debuggers are fundamental to productivity. However, in contrast to the significant attention given to mechanisms and tools for defining language extensions, comparatively few approaches have been proposed for the efficient construction of such development environments for domain-specific languages. In some cases ad-hoc editors, debuggers, analyzers, etc. have been developed from scratch. However, this approach is time consuming, error prone, hard to maintain, and usually not scalable to a variety of language extensions.

A more attractive alternative, at least conceptually, is to reuse the tools available for the target language, such as its debuggers or analyzers. This can in principle save much implementation effort, in the same way in which the source-to-source approach leverages the implementation of the target language to support the domain-specific extensions. However, the downside of this approach is that these tools will obviously communicate with the programmer in terms of the target language. Since a good part of the syntactic structure of the input source code is typically lost in the transformation process, these messages and debugger steps in terms of the target language are often not easy to relate with the source level and then the target language tools are not really useful for their intended purposes. For example, a debugging trace may display auxiliary calls, temporary variables, and obscure data encodings, with no trivial relation with the control or data domain at the source level. Much of that information is not only hard to read, but in most cases it should be invisible to the programmer or domain expert, who should not be forced to understand how the language at the source level is embedded in the supporting language.

In this paper, we propose a method for recovering symbolically the source of particular translations (that is, reversing them and providing an unexpanded view when required) in order to make target language level development tools useful in the presence of language extensions. Our solution is presented in the context of Ciao [8], which uses a powerful language extension mechanism for supporting several paradigms and (sub-)languages. We augment this extension mechanism with support for symbolic annotations that enable the recovery of the source code information at the target level. As an example application, we 
use these annotations to parameterize the Ciao interactive debugger, so that it displays domain-specific information, instead of plain Prolog goals. Our approach requires only very small modifications in the debugger and the compiler, which can still handle other language extensions in the usual way.

The paper is organized as follows: Section 2 presents a concrete extension mechanism and illustrates the limitations of the traditional translation approach in our context. Section 3 presents our approach to unexpansion, and guidelines for instrumenting language extensions so that the intervening translations can be reversed as needed into their input source code. Section 4 presents the application of the approach to the case of debuggers. Finally, Section 5 presents related work and Section 6 concludes and suggests some future work.

\section{Language extensions and their limitations}

We present a concrete language extension mechanism based on translations (the one implemented in the Ciao language) and then illustrate the limitations of the traditional translation-based extension approach in our context. In Ciao [8], language extensions are implemented through packages [2], which encapsulate syntactic extensions for the input language, translation rules for code generation to support new semantics, and the necessary run-time code. Packages are separated into compile-time and run-time parts. The compile-time parts (termed compilation modules) are only invoked during compilation, and are not included in executables, since they are not necessary during execution. On the other hand, the run-time parts are only required for execution and are consequently included in executables. This phase distinction has a number of practical advantages, including obviously the reduction of executable sizes.

More formally, let us assume that an extension for some language denoted as $\mathcal{L}_{e}$ is defined by the package $P k g M o d_{e}$, and that the compiler passes include

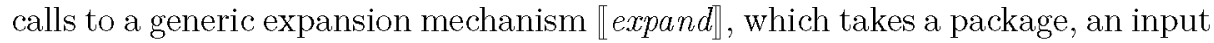
program in the source language, and generates a program in the target language $\mathcal{L}$. That is, given $\llbracket$ expand $\rrbracket_{e}=\llbracket$ expand $\rrbracket\left(\right.$ PkgMod $\left._{e}\right)$, for a program $P_{e} \in \mathcal{L}_{e}$ we can obtain the expanded version $\llbracket$ expand $\rrbracket_{e}\left(P_{e}\right)=P \in \mathcal{L}$. Note that in practice, Ciao contains finely grained translation hooks, which allow a better integration with the module system and the composition of translations [13]. This level of detail is not necessary for the scope of this paper, and thus, for the sake of simplicity, the expansion will work on whole programs at a time.

Functional notation. We illustrate the translation process in Ciao with an example from the functional notation package [3]. This package extends the language with functional-like syntax for relations. Informally, this extension allows including terms with predicate symbols as part of data terms, while interpreting them as predicate calls with an implicit last argument. It also allows defining clauses in functional style where the last argument is separated by a := symbol (as well as other functionalities, such as expanding goals in the last argument after the body). The translation can be abstractly specified as a collection of 
Source code (functional notation)

$f(X):=X<42 ?$

$(\mathrm{k}(\mathrm{l}(\mathrm{m}(\mathrm{X}))) * 3)$

1000 .

$\mathrm{k}(\mathrm{X}):=\mathrm{X}+1$.

$I(X):=X-2$.

$\mathrm{m}(\mathrm{X}):=\mathrm{X}$.
Target code (plain Prolog)

$f(X$, Res $):-X<42, \quad !$, $m(X, M), l(X, L), k(X, K)$, $\mathrm{T}$ is $\mathrm{K} * 3$, $\mathrm{T}=$ Res.

$f(X, 1000)$.

$\mathrm{k}(\mathrm{X}, \operatorname{Res}):-$ Res is $\mathrm{X}+1$.

$l(X, R e s):-$ Res is $X-2$.

$m(X, X)$.

Fig. 1. Example translation for functional notation

rewrite rules such as:

$$
\begin{array}{rrrl}
\text { (Clauses) } & \operatorname{tr} \llbracket p(\bar{a}):=C:-B \rrbracket & =\left(p^{\prime}(\bar{v}, T):-\bar{v}=\bar{a}, B, T=C\right) \\
\text { (Calls) } & \operatorname{tr} \llbracket q(\ldots p(\bar{a}) \ldots) \rrbracket & =\left(p^{\prime}(\bar{a}, T), q(\ldots T \ldots)\right)
\end{array}
$$

The first rule describes the meaning of a clause in functional notation, where $p^{\prime}$ is the predicate in plain syntax corresponding to the definition of $p$ in functional notation (i.e., using :=). The second rule must be applied using a leftmostinnermost strategy for every $p$ function symbol that appears in the goal $q$, where $T$ is a new variable (skipping higher-order terms). If SLD resolution is used, the evaluation order corresponds to eager, call-by-value evaluation (but lazy evaluation is possible and shown in [3]). We refer to the actual implementation later in this section.

Example 1. In Figure 1 we show an example program that defines a predicate $\mathrm{f} / 2$ in functional notation and its translation into plain Prolog code. Its body contains nested calls to $\mathrm{k} / 2,1 / 2, \mathrm{~m} / 2$, and also syntactic sugar for a conditional (if-then-else) construct (using the syntax: CondGoal? ThenExpr $\mid$ ElseExpr).

Forgetful Translations and Loss of Symbolic Information. Both the standard compilation and the translations for language extensions are typically focused on implementing some precise semantics during execution. That is, the correctness of the translation guarantees that for all programs $P_{e} \in \mathcal{L}_{e}$, the expected semantics $\llbracket$ exec $\rrbracket_{e}$ for that language can be described in terms of a program $P \in \mathcal{L}$ and its corresponding execution mechanism exec $\rrbracket$ That is, for all $P_{e} \in \mathcal{L}_{e}$ there exists a $P=\llbracket \operatorname{expand} \rrbracket_{e}\left(P_{e}\right)$ so that $\llbracket \operatorname{exec} \rrbracket_{e}\left(P_{e}\right)=\llbracket \operatorname{exec} \rrbracket(P)$.

Most of the time, symbolic information at the source level is lost, since it is not necessary at run time. In particular, such information removal and loss of structure is necessary to perform important program optimizations (e.g.,assigning some variables to registers without needing to keep the symbolic name, its relation to other variables in the same scope, etc.). When programs are not necessarily executed, but manipulated at a symbolic level, the translation-based approach is no longer valid on its own. For example, assume a simple debugger that interprets the source and allows the user to inspect variable values at each 


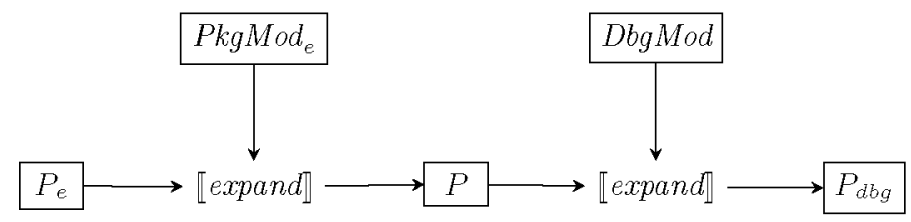

Fig. 2. The translation process and application of the standard debugger.

program point interactively. In this case the translation, as a program transformation, must preserve not only the input/output behaviour but also some other observable features (such as line numbers or variable names).

In order to explore the particular case of debuggers more closely, Figure 2 illustrates the translation process of a source program, using a compilation module $\mathrm{PkgMod}_{e}$ containing the translation rules for extension $e$. If the developer asks the Ciao interpreter to debug this program, further instrumentation is applied that is also defined in part as a language extension, DbgMod in Figure 2; this instrumentation customizes the code by encapsulating it into a predicate that specifies whether a part of the code is spy-able or not. The following example illustrates in a concrete case the limitations of this process.

Example 2 (Interactive debugging). Consider the code and transformation of Example 1. If the target-level debugger is used without any other provision, following the process of Figure 2, debugging a call to $f(3, \mathrm{~T})$ amounts to debugging its translation, as illustrated in the trace of Figure 3 (the exit calls are omitted in order to save space). The problem of this trace is twofold: first, the interactive debugging does not make explicit the actual source-level predicate that is currently being tested. Second, understanding the trace forces the developer to make the mental effort of analyzing the debugged data and mapping it back to the source code. This effort increases if the source code contains operators that do not exist on the target (Prolog) side. The first case can be easily overcome when operator definitions are shared, e.g.,using a graphical editor and catching the operator with the line number and the occurence number of the call. However, the second case implies remembering the mapping between the source and the target operator. Furthermore, things get even more tedious and intricate

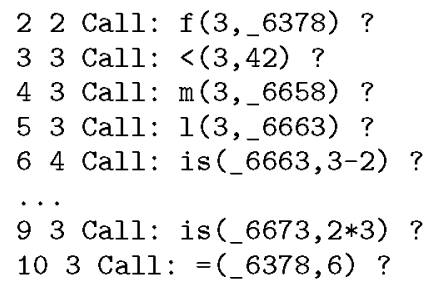

Fig. 3. Excerpt of the display of the interactive debugger. 
when one instruction in the source language is translated into a composition of goals.

\section{Building reversible extensions}

In this section we provide an informal definition of unexpansion with respect to a language extension. We then present guidelines in order to instrument a compilation module for such a language extension. The purpose of this instrumentation is to drive the process of reconstructing a program in terms of the language extension (or source language) in which the program is written. Through this mechanism, a language extension can be made reversible. To illustrate our objective, we apply the guidelines and parameterize one of the translation rules used in the functional notation extension.

\subsection{A correspondence between expansion, unexpansion, and observers}

We use the term unexpansion to designate the inverse of the expansion $\llbracket$ expand $\rrbracket_{e}$, that is, the recovering of the original $P_{e}$ source program from $P$. Unfortunately, this inverse is rarely a one-to-one mapping. For example, $f(3, T)$ in $\mathcal{L}$ corresponds to both $T=f(3)$ and $f(3, T)$ (with $f / 1$ using functional notation). For another example, a clause can either be translated in one or many clauses, as depicted in Figure 1 for $f$ in functional notation.

Not existing a unique solution can be confusing for the user and impractical for automatic transformations. However, the most important use of unexpansion in our context is to observe the behavior of only certain program aspects at the source language level. In this case, unexpansion seems more treatable. For that purpose we define the term observer accordingly: an observer is an interface that provides some specific source-level information about a particular program. The observer can be either static or dynamic. Specifically, we can consider as observers monitors (e.g.,interactive debuggers, tracers, and profilers) for dynamic observation, and verifiers (e.g.,static analyzers and model checkers) for static observation. Thus, a source-level view may correspond to the current instruction being invoked in an interactive debugger, or to a trace of the memory state, in a tracer, or perhaps the dependencies between the program variables, in a static analyzer, all of them represented in terms of the source language abstractions.

The correspondance between expansion and unexpansion, in the context of an observer, is sketched in Figure 4. We assume that we have observers $\mathrm{Obs}_{e}(i)$ and $\mathrm{Obs}(i)$ for the source and target languages, respectively. We denote by $i$ some particular observable aspect and by $V$ the aspect (e.g., "line numbers" and an integer). On the left diagram we depict the impossibility of getting information

at the $\mathcal{L}_{e}$ level in general. To provide the programmer with source-level observers, our approach relies on extending the expansion $\left(\llbracket\right.$ expand $\left.d \rrbracket_{e}^{\text {sym }}\right)$ with additional symbolic information (which can be significantly smaller than the sources). Then, observers $\mathrm{Obs}^{\mathrm{sym}}(i)$ can retrieve $V$ (e.g., a single number encoding the row and 

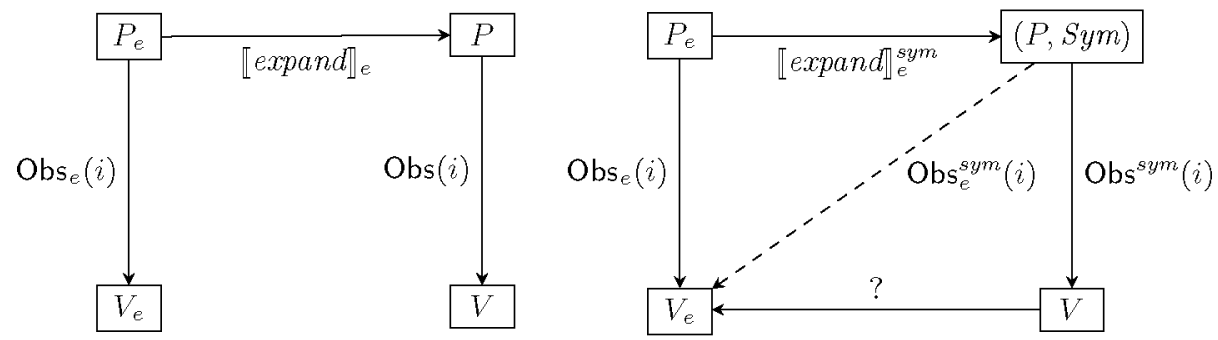

Fig. 4. Observation problem at the source level (left); Observation using symbolic information (right).

columns) and map it back to $V_{e}$ (e.g.,the row and columns). This composition provides an effective $\mathrm{Obs}_{e}^{\text {sym }}(i)$.

We now propose guidelines for easily instrumenting the translation module of a language extension, in such a way that observers can be parameterized with respect to this instrumentation.

\subsection{Instrumentation of a compilation module}

Instrumenting a compilation module involves annotating its translation rules with source code information that can then be used by an observer (i.e., the debugger in our application example). We illustrate the instrumentation process on the functional extension example.

Guidelines. The first step in making a language extension reversible is to determine which parts of the source code need to be kept available in the expansion process. The second step is to determine how and where to propagate this information, so that it can be accessed whenever the developer requires observation during program execution. The third step is to determine the representation of the observable data.

Event and data analysis. What events do we want to observe? What do we want to observe about them? These selections should be useful for following the control flow and state changes during program execution. For example, in a $\lambda$-calculus-like language, the definition and the application of a function are two of the key elements to follow in order to debug a program [16]. As another example, in a goal involving expressions in functional notation, the debugger must be aware of which positions correspond to data terms and which positions to predicate calls.

Decomposition. How is a source statement decomposed into target code? The answer to this question implies in part how the data that we want to observe should be propagated. For example, while the generic debugger may step through 
a number of target-level statements, a source-specific debugger may have to consider a single source statement as corresponding to all those steps. This applies for example in the conditional statement C ? A | B of the functional notation, where $\mathrm{A}$ is translated into an (at least) two-goal target code segment.

Representation. How should the data to be observed be represented? In a purely syntactic extension, data always represents elements of the concrete syntax. Nevertheless, it is interesting to consider this question when displaying the runtime context, such as the state of the memory, for semantic extensions.

For example, in a $\operatorname{CLP}\{\mathrm{Q}, \mathrm{R}\}$ extension, variables are bound at run-time to complex terms attached to attributed variables which reflect the internal, lowlevel representation of the constraint store, while what the programmer would like to see is a symbolic representation of the constraints among the variables in the source constraint language.

Instrumentation in action. To instrument the translation rules we propose to annotate the target parameter of each rule (i.e., the argument in which the code generated by the translation is returned). This annotation (which we call the meta-annotation) is defined as a macro which provides the symbolic information to drive the process of recovering source code data within the observer. It may contain any data written in a prolog syntax, enabling to recover some source level information.

For example, such annotation could be a list of variables and a function enabling to recover their value in the source level notation from the target context (its environment and store), or a single string to be displayed at the observer's output at run time.

We currently distinguish two types of meta-annotations: the \$clause_info annotation, which is wrapped around target clauses, and the \$goal_info metaannotation, which is wrapped around target goals. The purpose of each of these meta-annotations is to gather symbolic information to recover a source-level statement or a source-level call, respectively. Additionally, this distinction enables to handle clauses and goals properly, in particular to retrieve their location in source modules.

A meta-annotation takes two arguments: the first argument is the wrapped element (i.e., the original clause or goal(s) generated by the transformation), and the second one provides symbolic information enabling to recover an "observable" representation of the wrapped element, according to what the extension designer wants the programmer to observe. We illustrate this annotation process with Example 3.

Example 3. Let us consider the translation rule for clause declarations in the functional notation package. This rule, named defunc, translates such clause declarations into a set of clauses:

defunc ( (FuncHead := FuncValOpts), Clauses) :-

FuncValOpts $=($ FuncVall | FuncValR $)$, , 


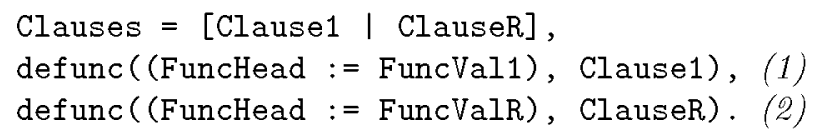

The FuncHead part on the left corresponds to a predicate declaration; the FuncValOpts part on the right corresponds to goal invocations (this results from the data analysis guideline). Notice that the declaration is decomposed into many goals (marked (1) and (2)) if the I operator appears inside its right part. Therefore, the translation needs to be adapted slightly, in order to indicate to the debugger that the declaration is to be treated as a single one. As illustrated in Example 4 below, the resulting adaptation amounts to creating an intermediate predicate (defunc_rec, not really necessary in this simple case), and to annotating the def unc rule (this results from the decomposition guideline). Note that the \$clause_info wrapper effectively groups all the clauses into which the definition is expanded, and this can be detected by the observer which will then treat it as a single clause.

The symbolic information attached to the annotation is represented by the contents of variable SI. This variable is handled by an observer, according to the nature of the program view it aims to provide. For example, line numbers, variables or function names can be attached to it. It can even be left as a free variable, in cases where the observer can automatically retrieve the information.

This approach based on meta-information enables us to envision a range of program views, from simple syntax recovery to high-level representation of analysis results: annotations can be enriched with source-specific procedures to handle various representations of the target program, enabling different instantiations of the meta-annotation variable. They can even hold procedures that perform advanced computations parameterized with the symbolic information (e.g.,counting the number of times a function is invoked).

Example 4. The instrumentation of the translation rule for declarations in functional notation writes as follows:

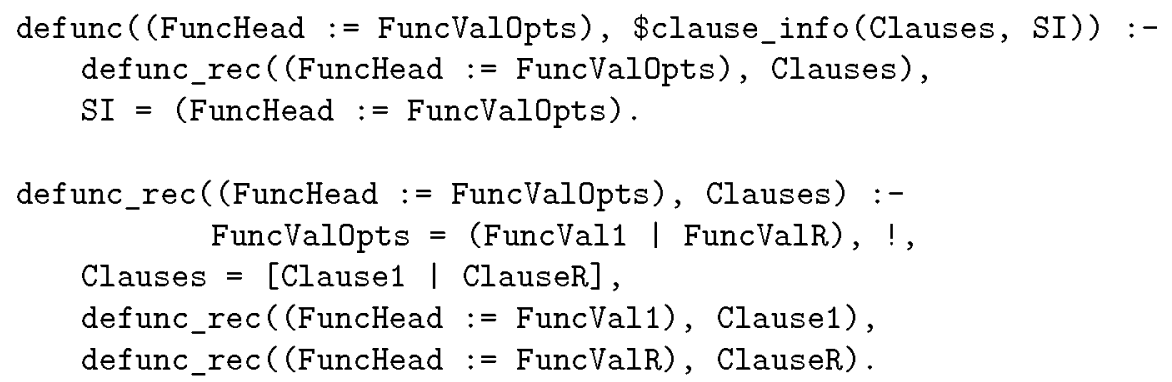

The same instrumentation method applies to goals, as outlined in the schema of Figure 5, which depicts a declaration of the form $\mathrm{f}(\mathrm{X}):=$ Cond ? $B_{1} \mid B_{2}$. In this figure, the variable names $S x$ correspond to symbolic information for some program elements (like goals or clauses), and the expressions $\operatorname{tr} \llbracket x \rrbracket$ correspond to 


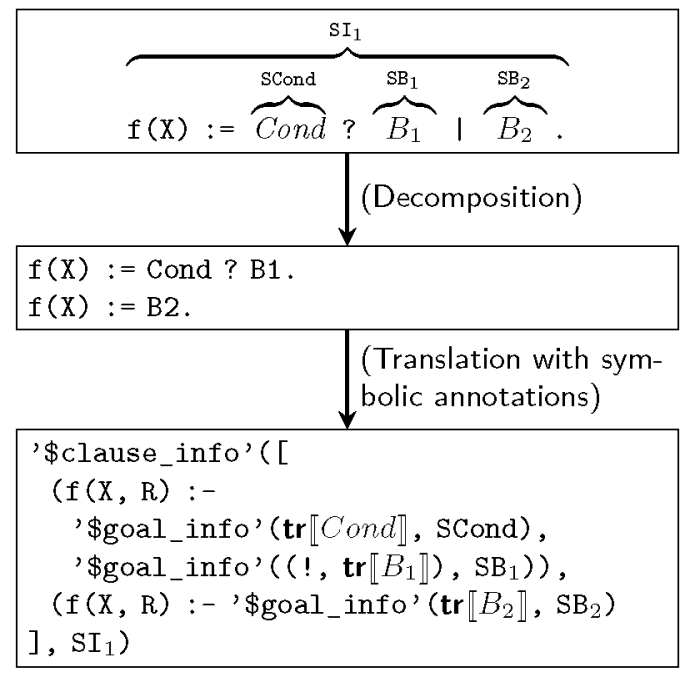

Fig. 5. Instrumented translation of a clause in functional notation.

a translation of the term $x$. To avoid the overloading of the compilation module with annotations, symbolic information can be stored in a specific table.

\section{Application to the interactive debugger}

We now illustrate the use of a reversible language extension to parameterize the generic interactive debugger of Ciao. We describe the modifications performed on the compiler and on the debugger, and show the resulting source-level trace for our initial example of Figure 1.

\subsection{Implementation details}

The overall process of making program behavior observable at the source level through a debugger and reversible expansion is depicted in Figure 6.

The compiler is responsible for applying both the debugger compilation module and the source language compilation module. Prior to applying the translation rules, it extracts the elements corresponding to sentences, clauses, and goals. During this step, information to locate the source program instructions are saved, such as the module name, the line numbers for sentences, and the name of the goal being called. Then, sentences, clauses, and goals are translated according to the specifications of the corresponding compilation module. To enable the handling of the term_info meta-annotations in Ciao, the translation step (represented by the translator box in Figure 6) of the compiler needs to be customized. This is done by performing an extraction step (represented by the extractor box in Figure 6, right part) that modifies the translation process when a meta-annotation is encountered. 


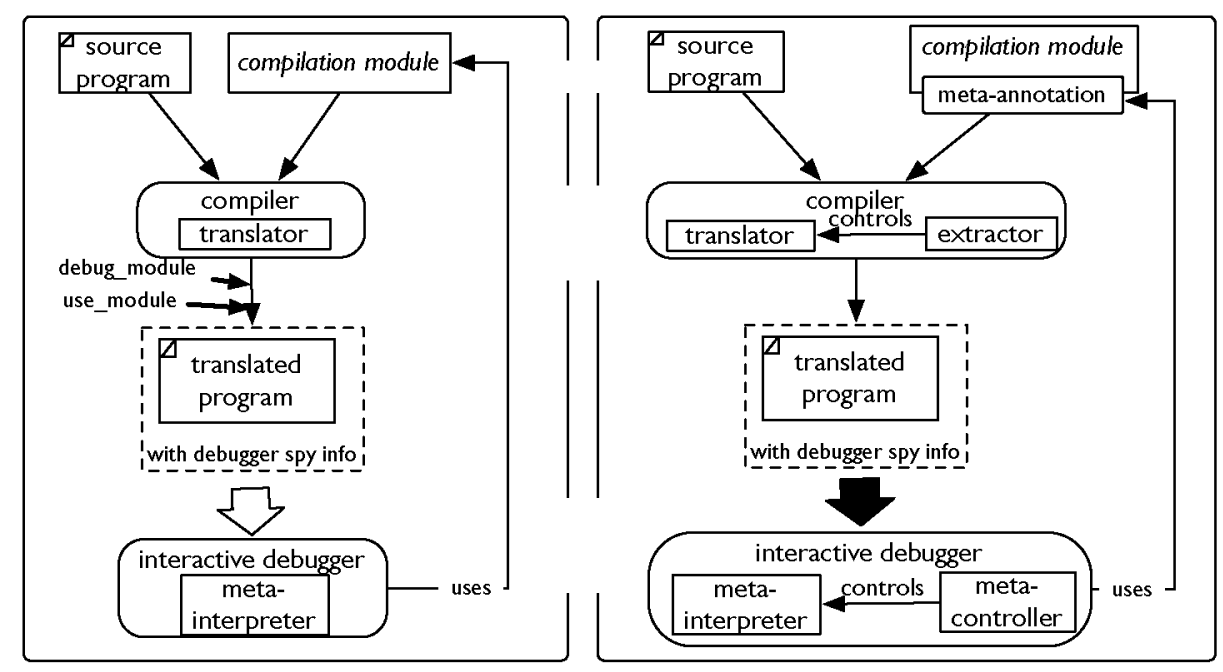

Fig. 6. Implementation: original (left) vs. customized (right) infrastructure.

In the case of the debugger, the required symbolic information corresponds to a source node $(e . g, k(\mathrm{X}):=\mathrm{X}+1$ as in Figure 1). As a result, the extraction process consists solely of storing each source node (either a clause or a goal) before its expansion.

Once the source-level information is extracted and mapped to the appropriate target term (or composition of target terms, cf. the guidelines in Section 3), it is interpreted by the debugger. To step through the source code instead of the target code, the debugger is equipped with a meta-controller, which checks the presence of a meta-information call at the level of the translated program, and displays a trace step accordingly. In particular, it is responsible for locating the name of the target goal in the source nodes corresponding to this goal. Since the compiler provides the source code information as a Prolog term, this localization is straightforward. When a goal invoked in the debugger has not been annotated (with \$goal_info), the meta-controller looks into the last \$clause_info metaannotation, and looks for the name of this goal inside this meta-annotation. Otherwise, the standard, expanded debug information is displayed.

\subsection{Source-level tracing: the functional example revisited}

With this instrumentation, Example 1 is now debugged in source code terms, as illustrated in Figure 7. Note that the debugger now displays the complete declaration (see second line) defining $f$, instead of a single part of a clause (see the second line in Example 1). When a function evaluation returns a value (which is the case of all the functions $f / 1, k / 1,1 / 1, m / 1$ ), intermediate unifications are performed by the generic debugger. When the debugger is instrumented with a meta controller (i.e., the handler of meta-annotations), these unification steps are 
ignored (skipped over), since they have no representation in the original source code.

\section{Related Work}

There exist frameworks and generative approaches that facilitate the development of DSL tools for programming, including debuggers $[6,19]$. For example, the Eclipse Integrated Development Environment [6], provides an API and an underlying framework that can greatly help in the development of a debugger [5]. Emacs is another example of such environments, with facilities in the same line as Eclipse. However, these tools are large and have a significant learning curve, and, more importantly, their facilities are centered more around the graphical navigation of the source code and interfacing with a command-line debugger, while the focus of our work is on bridging syntactic or semantic aspects between two sides of a translation, within such a command-line debugger. In that sense our work is complementary to (and in practice combines well with) the facilities in Eclipse, Emacs, and related environments. Generative approaches have been suggested (e.g.,based on aspect weaving into the language grammar [21]) in order to reduce developer burden when using intricate APIs.

However, none of these approaches provide a methodology for developing reliable and maintainable debuggers. As a result, the development of debuggers has remained difficult, inciting DSL tool developers to implement ad-hoc solutions, through extension-specific modifications and adaptations of the debugger code. For example, SWI-Prolog includes a graphical debugger for Prolog with built-in support for DCGs and Logtalk programs [20]. As mentioned in the introduction, this approach results in useful debuggers but which are specific to concrete extensions. As a result, they have to be modified again for other transformations.

Our objective has been to develop a more general approach, which we have illustrated by applying the same methodology to several extensions including functional notation, DCGs, and CLP $\{\mathrm{Q}, \mathrm{R}\}$.

Lindeman et al. [11] have proposed recently a declarative approach to defining debuggers. To this end, they use SDF [18], a rewriting system, to instrument the

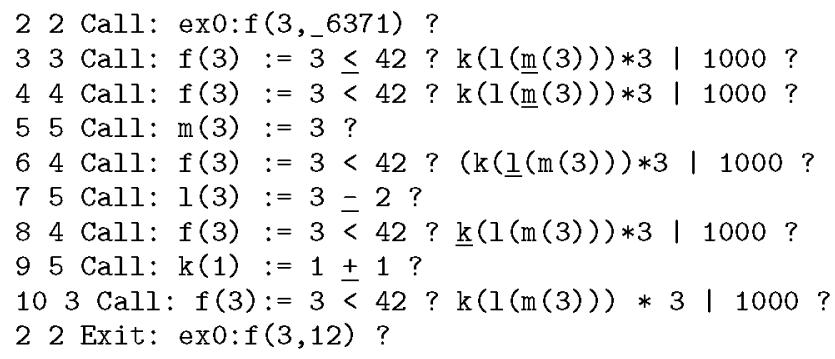

Fig. 7. An excerpt of the debugger trace, customized with source information. 
abstract syntax tree with debugging annotations. However, it does not seem obvious that their approach could be applied to other observer tools. Indeed, instrumentation is achieved by providing debugger-specific information, in the form of events. In contrast, our instrumentation process makes it possible to easily add and handle different kinds of meta-information.

Unexpansion and decompilation only differ in the hypothesis used in decompilation: that the original source code may not be available. It is interesting however to compare to existing related decompilation approaches. Bowen [1] proposes a compilation process from Prolog to object code which makes it possible to define decompilation as an inverse call to compilation, provided some reordering of calls is performed. Gomez et al. [7] also propose a decompilation process for Java based on partial evaluation. However, these approaches have not been designed to be applicable to a large class of different language extensions. More generally, while it is in theory possible (although predictably hard with current technology) to implement fully reversible transformations, this approach runs into the problem that such inversions are non-deterministic in general, in the sense that a given target code can be generated from multiple source texts. Presenting the programmer with a different code that what is in the source program could be even more confusing that debugging the target code directly.

More similar to our solution is the approach of Tratt [17], which also targets language extensions, and where source information is injected into the abstract syntax tree of the source program. This information is exploited to report errors in terms of the language extension. However, they only discuss how to inject such information in the syntax tree, and do not explain how to use this information when building or adapting tools.

The macro-expansion passing style [4] approach makes it possible to easily implement observers. Our approach differs from this one in the reliance on the existing generic debugger (Ciao's in our examples), and concentrates instead on what changes are required in the debugger and the extension framework in order to handle meta-information for unexpansion in a way that is independent from the concrete language extension.

As a conclusion, we believe that our process proposal could be extended to other Prologs, as the meta-annotations enable to hold symbolic information that is made available in most Prolog compilers, e.g., line numbers or variable names.

\section{Conclusion and future work}

We have presented a generic approach that enables a debugger for a target language to display trace information in terms of the language extension in which a source program is written, using the Ciao debugger as an example. The proposed approach is based on an extension of the usual mechanisms for term expansion, and in particular of their modular implementation in Ciao through packages. Specifically, we define a methodology for making relevant parts of the source text and other characteristics at the target level by enriching the translation rules. We have shown that the compiler and the debugger require 
only small adaptations in order to take this mechanism into account and that these adaptations are generic in the sense that while the transformation rules are of course specific to the extension, the compiler and debugger themselves do not require further modification, for what is arguably a usefully large class of extensions. In particular, in the paper we have illustrated this approach by applying it on the functional notation. In the system, we have successfully applied it also to the DCG and CLP $\{\mathrm{Q}, \mathrm{R}\}$ constraint packages.

In future work, we plan to extend the flexibility of the approach by enriching the annotations, and being able to provide different annotations for different purposes. Also, we feel that this initial work on augmenting the language extension mechanism already provides us gives with the basis for adapting the Ciao pre-processor so that for example errors, warnings, and other reports are made in terms of the source, domain-specific language, for different extensions, without requiring further modification of the pre-processor itself. The same would apply of course to the auto-documenter.

Finally, we could leverage Kishon et al.'s framework [10] to check the soundness of our approach with regard to the intended semantics of a language extension. Doing so would also enable to show the equivalence between the behavior of an ad-hoc source level debugger and our customization of the target level debugger.

\section{Acknowledgments}

The research leading to these results has received funding from the Madrid Regional Government under CM project P2009/TIC/1465 (PROMETIDOS), and the Spanish Ministry of Economy and Competitiveness under project TIN2008-05624 DOVES.

\section{References}

1. J. P. Bowen. From programs to object code and back again using logic programming: Compilation and decompilation. Journal Of Software Maintenance Research And Practice, 5(4):205-234, 1993.

2. D. Cabeza and M. Hermenegildo. A New Module System for Prolog. In International Conference on Computational Logic, CL2000, number 1861 in LNAI, pages 131-148. Springer-Verlag, July 2000.

3. A. Casas, D. Cabeza, and M. Hermenegildo. A Syntactic Approach to Combining Functional Notation, Lazy Evaluation and Higher-Order in LP Systems. In The 8th International Symposium on Functional and Logic Programming (FLOPS'06), pages 142-162, Fuji Susono (Japan), April 2006.

4. R. K. Dybvig, D. P. Friedman, and C. T. Haynes. Expansion-passing style: A general macro mechanism. Lisp and Symbolic Computation, 1(1):53-75, 1988.

5. Eclipse. How to write an Eclipse debugger. http://ww. eclipse.org/articles/ Article-Debugger/how-to.html.

6. ECRC. Eclipse User's Guide. European Computer Research Center, 1993. 
7. M. Gómez-Zamalloa, E. Albert, and G. Puebla. Decompilation of Java Bytecode to Prolog by Partial Evaluation. Information and Software Technology, 51(10):14091427, October 2009.

8. M. V. Hermenegildo, F. Bueno, M. Carro, P. López, E. Mera, J. Morales, and G. Puebla. An Overview of Ciao and its Design Philosophy. Theory and Practice of Logic Programming, 12(1-2):219-252, January 2012. http://arxiv.org/abs/1102.5497.

9. P. Hudak. Building domain-specific embedded languages. ACM Comput. Surv., 28(4es):196, 1996.

10. A. Kishon, P. Hudak, and C. Consel. Monitoring semantics: A formal framework for specifying, implementing, and reasoning about execution monitors. In PLDI, pages $338-352,1991$.

11. R. T. Lindeman, L. C. Kats, and E. Visser. Declaratively defining domain-specific language debuggers. In Proceedings of the 10th ACM international conference on Generative programming and component engineering, GPCE '11, pages 127-136, New York, NY, USA, 2011. ACM

12. M. Mernik, J. Heering, and A. M. Sloane. When and how to develop domainspecific languages. ACM Comput. Surv., 37(4):316-344, Dec. 2005.

13. J. F. Morales, M. V. Hermenegildo, and R. Haemmerlé. Modular Extensions for Modular (Logic) Languages. In 21th International Symposium on Logic-Based Program Synthesis and Transformation (LOPSTR'11), Odense, Denmark, July 2011. To appear.

14. N. Nystrom, M. R. Clarkson, and A. C. Myers. Polyglot: An extensible compiler framework for java. In Compiler Construction, pages 138-152, 2003.

15. F. Pereira and D. Warren. Definite clause grammars for language analysis - a survey of the formalism and a comparison with augmented transition networks. Artificial Intelligence, 13:231-278, 1980.

16. A. P. Tolmach and A. W. Appel. A debugger for standard ml. J. Funct. Program., $5(2): 155-200,1995$.

17. L. Tratt. Domain specific language implementation via compile-time metaprogramming. ACM Trans. Program. Lang. Syst., 30(6):31:1-31:40, Oct. 2008.

18. M. van den Brand, A. van Deursen, J. Heering, H. A. de Jong, M. de Jonge, T. Kuipers, P. Klint, L. Moonen, P. A. Olivier, J. Scheerder, J. J. Vinju, E. Visser, and J. Visser. The asf+sdf meta-environment: A component-based language development environment. In Compiler Construction, pages 365-370, 2001.

19. M. G. J. van den Brand, B. Cornelissen, P. A. Olivier, and J. J. Vinju. Tide: A generic debugging framework - tool demonstration - Electron. Notes Theor. Comput. Sci., 141(4):161-165, Dec. 2005.

20. J. Wielemaker. SWI-prolog - source-level debugger. http://www.swi-prolog. org/gtrace.html.

21. H. Wu, J. Gray, S. Roychoudhury, and M. Mernik. Weaving a debugging aspect into domain-specific language grammars. In Proceedings of the 2005 ACM symposium on Applied computing, SAC '05, pages 1370-1374, New York, NY, USA, 2005. ACM. 